\title{
Dimerization of Carboxylic Acids: An Equation of State Approach
}

Tsivintzelis, loannis; Kontogeorgis, Georgios; Panayiotou, Costas

Published in:

Journal of Physical Chemistry Part B: Condensed Matter, Materials, Surfaces, Interfaces \& Biophysical

Link to article, DOI:

10.1021/acs.jpcb.6b10652

Publication date:

2017

Document Version

Peer reviewed version

Link back to DTU Orbit

Citation (APA):

Tsivintzelis, I., Kontogeorgis, G., \& Panayiotou, C. (2017). Dimerization of Carboxylic Acids: An Equation of State Approach. Journal of Physical Chemistry Part B: Condensed Matter, Materials, Surfaces, Interfaces \& Biophysical, 121(9), 2153-2163. https://doi.org/10.1021/acs.jpcb.6b10652

\section{General rights}

Copyright and moral rights for the publications made accessible in the public portal are retained by the authors and/or other copyright owners and it is a condition of accessing publications that users recognise and abide by the legal requirements associated with these rights.

- Users may download and print one copy of any publication from the public portal for the purpose of private study or research.

- You may not further distribute the material or use it for any profit-making activity or commercial gain

- You may freely distribute the URL identifying the publication in the public portal 


\title{
On the Dimerization of Carboxylic Acids: An Equation of State Approach
}

\author{
Ioannis Tsivintzelis*, ${ }^{*}$, Georgios M. Kontogeorgis ${ }^{2}$ and Costas Panayiotou ${ }^{1}$ \\ ${ }^{1}$ Department of Chemical Engineering, Aristotle University of Thessaloniki, 54124 \\ Thessaloniki, Greece. \\ ${ }^{2}$ Center for Energy Resources Engineering (CERE), Department of Chemical and \\ Biochemical Engineering, Technical University of Denmark, DK-2800 Kgs. Lyngby, \\ Denmark.
}

*Corresponding author: Ioannis Tsivintzelis: E-mail: gtsivint@eng.auth.gr

\begin{abstract}
The association term of the Non-Random Hydrogen-Bonding theory, which is an equation of state model, is extended to describe the dimerization of carboxylic acids in binary mixtures with inert solvents and in systems of two different acids. Subsequently, the model is applied to describe the excess enthalpies and the vapor-liquid equilibrium of relevant binary mixtures containing low molecular weight organic acids. The model sheds light on the interplay of intermolecular interactions through the calculation of the various contributions to the mixing enthalpies, namely from hydrogen bonding and non-hydrogen bonding (dipolar, induced polar or dispersive) interactions. According to model predictions, the acid molecules are so strongly associated that the addition of inert solvents to carboxylic acids with small carbon numbers at ambient temperature does not dramatically alter their degree of association. Consequently, the observed endothermic dissolution process is mainly attributed to the hindering of polar interactions. Furthermore, upon mixing of two carboxylic acids, the rearrangement of hydrogen bonds due to the formation of cross associating species results in an insignificant contribution to the heats of mixing due to the rather constant dimerization enthalpy that is revealed by the available experimental data for low molecular weight compounds.
\end{abstract}




\section{Introduction}

The rational design of modern processes in chemical and pharmaceutical industries, as well as the great potential of new bioengineering applications, strengthened the need for accurate modeling of complex mixtures that often exhibit highly non-ideal solution behavior, such as aqueous systems, biological mixtures of aminoacids, proteins and other biomolecules, complex pharmaceutical systems, dyes, extractives and binders, gels, polymer solutions and blends. Hydrogen bonding dictates the phase behavior of most systems of such kind.

Carboxylic acids are among the groups of fluids with peculiar hydrogen bonding behavior and the accurate modeling of such fluid mixtures is important for many processes that include biological systems, but also traditional chemical applications, e.g the production of terephthalic acid. ${ }^{1,2}$ Such peculiar hydrogen bonding behavior arises from the formation of two hydrogen bonds between two acid molecules. Since the first bond is established, the formation of the second becomes more feasible (hydrogen bond cooperativity). The cooperativity in the establishment of the second bond results in the formation of stable dimers and, thus, the formation of linear oligomers is not favored. $3,4,5$

Accounting for hydrogen bonding through an equation of state approach is usually performed using thermodynamic models of two broad families, i.e. models that are based on the lattice fluid theory ${ }^{6-12}$, and all the variants of SAFT (Statistical Associating Fluid Theory) equation of state ${ }^{13-15}$, which are based on the perturbation theory of Wertheim. ${ }^{16-}$ 19

Such models were successfully applied in describing mixtures of hydrogen bonding fluids, such as aqueous solutions and systems with alcohols, amines or glycols, in many cases showing similar performance and limitations. ${ }^{20-22}$ In order to describe thermodynamic properties of organic acids, two association schemes are mainly used in the framework of the aforementioned thermodynamic models: the so called " $1 \mathrm{~A}$ " and " $2 \mathrm{~B}$ " association schemes. ${ }^{23-26}$ According to the first one, every acid molecule has one association site, positive or negative, which can be bonded with the relevant site of another acid molecule. The latter one allows the modelling of linear hydrogen bonded oligomers and is, thus, a simplified way of describing carboxylic acid systems. In the " $2 \mathrm{~B}$ " scheme, every acid molecule has two sites: one proton donor and one proton acceptor. Such 
association schemes, often result in successful description of the vapor-liquid equilibrium of carboxylic acid mixtures ${ }^{2,23-25}$, however, present serious limitations in aqueous solutions, the description of liquid - liquid equilibrium and more demanding calculations, such as the description of thermal properties. ${ }^{1,2}$ Only recently, the association term of both types of models was extended in order to describe the dimerization of organic acids in a more realistic way. ${ }^{27-30}$

Most often, in order to test the validity of a model, its predictions are compared solely to phase equilibrium data. ${ }^{3,20-26}$ Such kind of predictions are very useful in designing applications, where models are applied to predict the phase behavior of multicomponent mixtures. However, in some cases, the accurate prediction of thermal properties, such as the mixing enthalpies, is also important for a successful prediction of the energy demands. Nevertheless, the description of calorimetric properties of complex hydrogen bonding systems by thermodynamic models is a challenging task, even though such models may satisfactorily describe their phase behavior and/or their volumetric properties. Furthermore, the accurate prediction of thermal properties is the recommended way in order to check if the various intermolecular interactions are properly accounted for. In some cases, such type of calculations revealed interesting phenomena not easily shown with experimental techniques. For example, when the NRHB model was applied to predict the mixing enthalpies in mixtures of hydrocarbons with alkoxy-alcohols, which are typical fluids interacting via, both, inter- and intra-molecular hydrogen bonding, allowed the calculation of separate contributions (from various types of hydrogen bonding and from non-hydrogen bonding intermolecular interactions) to the total value of the property. ${ }^{31} \mathrm{It}$ was revealed that the value of the total and experimental measurable enthalpy of mixing was the sum of very different contributions, i.e. the endothermic contribution due to the decrease of the inter-molecular association and the exothermic contribution due to the simultaneous considerable increase of the intra-molecular association that occurs upon the addition of the inert solvent.

In this study, we present the formalism for describing the dimerization of organic acids in the framework of the Non Random Hydrogen Bonding theory, which is a lattice fluid thermodynamic model. ${ }^{11,12}$ The presented approach is based on the work of Panayiotou and coworkers $^{27}$ for pure fluids and here is extended to binary mixtures containing a carboxylic 
acid with an inert solvent or another organic acid. The Non Random Hydrogen Bonding Theory ${ }^{11,12}$ is a recent development of previous lattice models and so far has been applied to the description of phase equilibria of various types of mixtures with low or high molecular weight compounds $^{20-22}$ including pharmaceuticals ${ }^{32,33}$, hydrogen bonding polymer systems ${ }^{34}$ and ionic liquids. ${ }^{35}$

\section{Theory}

\subsection{The basics of the Non Random Hydrogen Bonding theory}

The Non-Random Hydrogen Bonding Theory (NRHB) is an equation of state model. ${ }^{11,12}$ It is an extension of previous successful compressible lattice models ${ }^{8-10}$, where holes are used to account for density variation as a result of temperature and pressure changes. It accounts explicitly for hydrogen bonding interactions and for the non-random distribution of empty and molecular sites in the quasi lattice.

Following the approach of Panayiotou and Sanchez ${ }^{9}$, the partition function of a system is factored into a "physical" term, $Q_{P}$, which accounts for all non-hydrogen bonding (dispersive and polar) interactions and a hydrogen-bonding (association) term, $Q_{H}$ :

$$
Q=Q_{P} Q_{H}
$$

Consequently, thermodynamic properties, such as the enthalpy or the free energy of a system can be divided into a physical and a hydrogen bonding (or association) contribution. For example the Gibbs free energy of a system is given by the following relation [9]:

$G=G_{P}+G_{H}$

In this study, we extend the formalism for describing the dimerization of organic acids. The development of the association term of the partition function, $Q_{H}$, and the resulting contributions to the equation of state and the chemical potentials due to the dimerization are presented in section 2.2. The former contribution in equation (1), due to physical interactions, is calculated using a combinatorial term based on Staverman approximation ${ }^{11,12}$ and a correction for non-randomness based on the quasi chemical theory of Guggenheim ${ }^{8}$. Such approach and the resulting equations of the model are adequately presented in literature ${ }^{11,12,36}$. Consequently, only some basic equations are briefly presented in section 2.3. 


\subsection{Association term}

In mixtures of carboxylic acids and inert solvents, dimers are the overwhelming majority of the association species at least at the vapor phase. In the following approach, we neglect any side reactions that may result in linear oligomers and we consider only dimerization, since this is the dominant hydrogen bonding behavior in the studied systems ${ }^{3,4,5}$. The presented approach is based on the formalism presented by Panayiotou and coworkers ${ }^{27}$ for pure fluids and here is extended to binary mixtures containing a carboxylic acid with an inert solvent or another organic acid.

\subsubsection{Mixtures of acids with inert solvents}

In a mixture of $N$ total molecules, which contains $N_{1}$ acid molecules and $N_{2}$ inert solvent molecules, let $N_{d m}$ be the number of acid dimers. The number of ways of selecting the $N_{d m}$ dimers is as follows:

$\Omega=\frac{N_{1} !}{\left(2 N_{d m}\right) !\left(N_{1}-2 N_{d m}\right) !}\left(2 N_{d m}-1\right)\left(2 N_{d m}-3\right) \ldots 1=\frac{N_{1} !}{\left(N_{1}-2 N_{d m}\right) ! N_{d m} ! 2^{N_{d m}}}$

The free energy change upon dimer $(d m)$ formation is ${ }^{27,37}$ :

$G^{d m}=E^{d m}+P V^{d m}-T S^{d m}$

Consequently, the hydrogen-bonding term of the partition function is ${ }^{27,37}$ :

$Q_{H}=\frac{N_{1} !}{\left(N_{1}-2 N_{d m}\right) ! N_{d m} ! 2^{N_{d m}}}\left(\frac{\tilde{\rho}}{r N}\right)^{N_{d m}} \exp \left(-\frac{N_{d m} G^{d m}}{R T}\right)$

where $\tilde{\rho}$ is the reduced density of the system and $r$ is the number of segments per molecule. The contribution to the free energy of the system due to dimerization is:

$G_{H}=-R T \ln Q_{H}$

The equilibrium number of dimers per mol of segments, $v_{d m}$, is obtained from the above equation through the free energy minimization condition: 


$$
\left(\frac{\partial G_{H}}{\partial N_{d m}}\right)_{T, P, N_{i}}=0
$$

which results in the following relation:

$v_{d m}=\frac{N_{d m}}{r N}=\frac{2 x_{1}+\frac{1}{K_{d m}}-\sqrt{\frac{1}{K_{d m}^{2}}+\frac{4 x_{1}}{K_{d m}}}}{4 r}$

where $x_{1}$ is the mole fraction of the acid in its mixture with the inert solvent and:

$$
K_{d m}=\frac{\tilde{\rho}}{r} \exp \left(\frac{-G^{d m}}{R T}\right)
$$

The hydrogen bonding contribution to the chemical potential of the acid is (for a binary system with an inert solvent):

$$
\frac{\mu_{1}^{H}}{R T}=\frac{1}{R T}\left(\frac{\partial G_{H}}{\partial N_{1}}\right)=r_{1} v_{d m}-\ln \frac{x_{1}}{x_{1}-2 r v_{d m}}
$$

The relevant contribution to the chemical potential of the inert compound is:

$\frac{\mu_{2}^{H}}{R T}=\frac{1}{R T}\left(\frac{\partial G_{H}}{\partial N_{2}}\right)=r_{2} v_{d m}$

\subsubsection{Mixtures of two acids}

In a mixture containing two acids of $N$ total molecules, the $N_{1}$ and $N_{2}$ of which are molecules of the first and the second compound, respectively, let $N_{d m}$ be the total number of dimers in the system, which include the $N_{11}$ and $N_{22}$ dimers consisting of molecules of the same kind (of type 1 and 2, respectively) and $N_{12}$ dimers consisting of molecules of different kind.

One can select the dimerized molecules of type 1 out of the $N_{l}$ acid molecules in 
$\omega_{1}=\frac{N_{1} !}{\left(2 N_{11}\right) ! N_{12} !\left(N_{1}-2 N_{11}-N_{12}\right) !}$

ways and the dimerized molecules of type 2 out of the $N_{2}$ acid molecules in

$\omega_{2}=\frac{N_{2} !}{\left(2 N_{22}\right) ! N_{12} !\left(N_{2}-2 N_{22}-N_{12}\right) !}$

ways. Between the $2 N_{11}$ molecules of type 1 , dimers can be formed in

$\omega_{3}=\left(2 N_{11}-1\right) \cdot\left(2 N_{11}-3\right) \cdot\left(2 N_{11}-5\right) \ldots \cdot(1)$

ways. Similarly, between the $2 N_{22}$ molecules of type 2 , dimers can be formed in

$$
\omega_{4}=\left(2 N_{22}-1\right) \cdot\left(2 N_{22}-3\right) \cdot\left(2 N_{22}-5\right) \ldots \cdot(1)
$$

ways, while between the $N_{12}$ molecules of type 1 and the $N_{12}$ molecules of type 2, dimers can be formed in $\omega_{5}=N_{12}$ ! ways. Consequently, the number of ways of selecting the $N_{d m}$ dimers is:

$\Omega=\omega_{1} \omega_{2} \omega_{3} \omega_{4} \omega_{5}=\frac{N_{1}}{N_{11} !\left(N_{1}-2 N_{11}-N_{12}\right) ! N_{12} ! 2^{N_{11}}} \cdot \frac{N_{2}}{N_{22} !\left(N_{2}-2 N_{22}-N_{12}\right) ! 2^{N_{22}}}$

The free energy change upon formation of an $i j$ dimer is:

$G_{i j}^{d m}=E_{i j}^{d m}+P V_{i j}^{d m}-T S_{i j}^{d m}$

Consequently, the hydrogen-bonding factor in the partition function becomes ${ }^{37}$ :

$$
Q_{H}=\Omega\left(\frac{\tilde{\rho}}{r N}\right)^{N_{d m}} \exp \left(-\frac{N_{11}^{d m} G_{11}^{d m}+N_{22}^{d m} G_{22}^{d m}+N_{12}^{d m} G_{12}^{d m}}{R T}\right)
$$

The contribution to the free energy of the system due to dimerization is given from equation (2), while the equilibrium number of dimers per mol of segments, $v_{11}, v_{22}, v_{12}$, is obtained from equations similar to equation (7), which result in the following relations: 


$$
\begin{aligned}
& \frac{2 v_{11}}{v_{10}{ }^{2}}=\tilde{\rho} \exp \left(-\frac{G_{11}^{d m}}{R T}\right) \\
& \frac{2 v_{22}}{v_{20}{ }^{2}}=\tilde{\rho} \exp \left(-\frac{G_{22}^{d m}}{R T}\right) \\
& \frac{v_{12}}{v_{10} v_{20}}=\tilde{\rho} \exp \left(-\frac{G_{12}^{d m}}{R T}\right)
\end{aligned}
$$

where $v_{11}=N_{11} / r N, v_{22}=N_{22} / r N, v_{12}=N_{12} / r N$ and

$v_{10}=\frac{x_{1}}{r}-2 v_{11}-v_{12}$

and

$$
v_{20}=\frac{x_{2}}{r}-2 v_{22}-v_{12}
$$

where $x_{1}$ and $x_{2}$ are the mole fractions of the two compounds. The contribution to the chemical potential is:

$$
\frac{\mu_{i}^{H}}{R T}=\frac{1}{R T}\left(\frac{\partial G_{H}}{\partial N_{i}}\right)_{T, P, N, j, \tilde{s},\left\{v_{i j}\right\}}=r_{i} v_{d m}-\ln \frac{x_{i}}{x_{i}-2 r v_{i i}-r v_{12}}
$$

where $\tilde{v}$ is the reduced molar volume. The total number of dimers per mol of segments in the system is:

$$
v_{d m}=v_{11}+v_{22}+v_{12}
$$

\subsection{The NRHB model}

According to the Non Random Hydrogen Bonding theory ${ }^{11,12}$, the molecules are distributed in a three-dimensional quasi lattice, with a lattice coordination number, $z$, which contains $N_{r}$ sites, $N_{0}$ of which are empty. Each molecule of type $i$ occupies $r_{i}$ sites and it is characterized by three scaling constants (pure fluid parameters) and one geometric, or surface-to-volume-ratio factor, $s$. The first two scaling constants, $\varepsilon_{h}^{*}$ and $\varepsilon_{s}^{*}$, are used for the estimation of the mean interaction energy per molecular segment, $\varepsilon^{*}$, according to the following equation:

$$
\varepsilon^{*}=\varepsilon_{h}^{*}+(T-298.15) \varepsilon_{s}^{*}
$$


while the third scaling constant, $v_{s p, 0}^{*}$, is used for the estimation of the close packed density, $\rho^{*}=1 / v_{s p}^{*}$, through the following equation:

$$
v_{s p}^{*}=v_{s p, 0}^{*}+(T-298.15) v_{s p, 1}^{*}
$$

The hard-core volume per segment, $v^{*}$, is constant and equal to $9.75 \mathrm{~cm}^{3} \mathrm{~mol}^{-1}$ for all fluids. Parameter $v_{s p, 1}^{*}$ in equation (27) is treated as a constant for a given homologous series. Finally, the shape factor, which is defined as the ratio of molecular surface to molecular volume, $s=q / r$, is calculated from the UNIFAC group contribution method. ${ }^{11}$ The equation of state of the model is given by ${ }^{11,12}$ :

$$
\tilde{P}+\tilde{T}\left[\ln (1-\tilde{\rho})-\tilde{\rho}\left(\sum_{i} \phi_{i} \frac{l_{i}}{r_{i}}-v_{d m}\right)-\frac{z}{2} \ln \left(1-\tilde{\rho}+\frac{q}{r} \tilde{\rho}\right)+\frac{z}{2} \ln \Gamma_{00}\right]=0
$$

while the chemical potential for component $i$ is calculated by the following equation ${ }^{12}$ :

$$
\begin{aligned}
& \frac{\mu_{i}}{R T}=\ln \frac{\phi_{i}}{\omega_{i} r_{i}}-r_{i} \sum_{j} \frac{\phi_{j} l_{j}}{r_{j}}+\ln \tilde{\rho}+r_{i}(\tilde{v}-1) \ln (1-\tilde{\rho})-\frac{z}{2} r_{i}\left[\tilde{v}-1+\frac{q_{i}}{r_{i}}\right] \\
& \ln \left[1-\tilde{\rho}+\frac{q}{r} \tilde{\rho}\right]+\frac{z q_{i}}{2}\left[\ln \Gamma_{i i}+\frac{r_{i}}{q_{i}}(\tilde{v}-1) \ln \Gamma_{00}\right]+r_{i} \frac{\tilde{P} \tilde{v}}{\tilde{T}}-\frac{q_{i}}{\tilde{T}_{i}}+\frac{\mu_{i}^{H}}{R T}
\end{aligned}
$$

where, $l_{i}$ and $\omega_{i}$ are characteristic quantities for each pure fluid, while $\varphi_{i}$ and $\theta_{i}$ are the site and the surface fraction of component $i$, respectively. Parameters $\Gamma_{o o}$ and $\Gamma_{i i}$ are nonrandom factors, which characterize the distribution of empty and molecular sites, respectively. Finally, the parameters $\tilde{T}=T / T^{*}, \widetilde{P}=P / P^{*}$ and $\tilde{v}\left(=1 / \tilde{\rho}=\rho^{*} / \rho\right)$ are the reduced temperature, pressure, and specific volume, respectively. The characteristic temperature, $T^{*}$, and pressure, $P^{*}$, are related to the mean intersegmental energy by:

$$
\varepsilon^{*}=R T^{*}=P^{*} v^{*}
$$

Detailed expressions for the calculation of such parameters can be found in previous studies. ${ }^{11,12,36}$

For dimerizing molecules, NRHB has three more parameters that are the energy, $E_{i j}^{d m}$, the volume, $V_{i j}^{d m}$, and the entropy change, $S_{i j}^{d m}$, for the formation of a dimer. However, usually the volume change is set equal to zero, so the number of the association parameters are reduced to two without compromising the performance of the model. ${ }^{11,12}$

\section{Results and Discussion}




\subsection{Pure Fluids}

One of the most important steps in applying equation of state models is the estimation of pure fluid parameters. Usually, this task is performed by adjusting model predictions to pure fluid experimental data, such as vapor pressures of liquids and saturated liquid densities. However, as already mentioned ${ }^{38,39}$, such procedure may result in more than one parameter sets that similarly describe the pure fluid properties, but not all of them can be successfully used for describing properties of binary and multicomponent mixtures. Regarding organic acids, such difficulty may be partially attributed to the dimerization of acids in the vapor phase, which strongly affects properties such as the vapor density or the heat of vaporization. For this reason, the use of experimental data for other properties, such as enthalpies of vaporization and vapor phase compressibility factors has been suggested. ${ }^{3,24,29}$ However, for all hydrogen bonding fluids, such difficulty mainly arises from the fact that the parameters that characterize the strength of the various intermolecular interactions (physical or hydrogen bonding), are highly correlated to each other. ${ }^{38,39}$ Consequently, the estimation of the association (dimerization) parameters $\left(E^{d m}\right.$ and $S^{d m}$ ) for carboxylic acids is probably the most important step towards the parametrization of such fluids. Hopefully, such parameters can be adopted from experimental spectroscopic, calorimetric and volumetric data or ab initio theoretical studies, due to the sound physical base of the model. However, in such an approach the most common problem is the diverse values for the association enthalpy that can be found in literature. ${ }^{4,40,41}$

Clague and Bernstein ${ }^{40}$, using IR spectroscopy, determined the enthalpy of dimerization of several low molecular aliphatic acids (with one up to five carbon atoms). They found that the dimerization enthalpy remains rather constant, independently of the alkyl group connected to the carboxylic group, since the electron releasing capacity of the alkyl-group increases the proton accepting ability of the carbonyl group, but simultaneously decreases the proton donating ability of the hydroxyl group. The two phenomena tend to cancel each other and, consequently, the (negative of the) enthalpy of dimerization in the vapor phase remains rather constant, around $60-65 \mathrm{~kJ} / \mathrm{mol}$. Pimentel and McClellan ${ }^{4}$, who collected data from several literature experimental studies, report that hydrogen bonding enthalpies around $30 \mathrm{~kJ}$ per mole of hydrogen bonds (consequently a dimerization enthalpy around $60 \mathrm{~kJ}$ per mole of dimers is a reasonable approximation) for vapor phase associated acid molecules. In aliphatic acids these values are almost constant regardless of the branching or the length (up to heptanoic acid) of the carbon chain. On the other hand, according to the authors, the most reliable data for liquid stearic acid gives $28 \mathrm{~kJ}$ per mole of hydrogen bonds, which is somewhat lower than the reported values for the association in the vapor phase. Murthy and $\mathrm{RaO}^{41}$ collected data from IR experimental studies and report values between $35-62 \mathrm{~kJ} \mathrm{~mol}^{-1}$ for acetic acid. 
Since the hydrogen parameters are of great importance, the NRHB association parameters for carboxylic acids were estimated first. In accordance with the aforementioned literature studies, the association (dimerization) parameters ( $E^{d m}$ and $S^{d m}$ ) of the NRHB theory were kept constant for low molecular weight carboxylic acids, while the association enthalpy was allowed to vary between 50 and $65 \mathrm{~kJ}$ per mole of dimers. According to a preliminary investigation, the optimum values of such model parameters were $52.5 \mathrm{~kJ} \mathrm{~mol}^{-1}$ and $46.2 \mathrm{~J} \mathrm{~mol}^{-1} \mathrm{~K}^{-1}$, for the association enthalpy and entropy, respectively. Subsequently, the rest three pure fluid parameters (scaling constants), $\varepsilon_{h}^{*}, \varepsilon_{s}^{*}$ ,$v_{s p, 0}^{*}$, were estimated, by fitting the predictions of the NRHB theory to the experimental data and keeping the association parameters equal to the aforementioned values. As mentioned above, the $v_{s p, 1}^{*}$ parameter (see equation (27)) is treated as a constant for a given homologous series and it was set equal to $-0.170 \cdot 10^{-3} \mathrm{~cm}^{3} \mathrm{~g}^{-1} \mathrm{~K}^{-1}$ for carboxylic acids.

Initially, the pure fluid scaling constants for acetic acid were estimated using data for saturated liquid densities and vapor pressures (DIPPR correlations ${ }^{42}$ ) as well as data for enthalpies of vaporization (experimental data included in DIPPR database ${ }^{42}$ ) and vapor phase compressibility factors ${ }^{43,44}$. For all other acids, saturated liquid densities, vapor pressures and enthalpies of vaporization were used (DIPPR correlations ${ }^{42}$ ). The estimated pure fluid parameters for the investigated acids are shown in Table 1 and some characteristic calculations are illustrated in Figures 1-4.

Table 1. NRHB pure fluid scaling constants for carboxylic acids and percentage average absolute deviations ${ }^{1}$ (\% AAD) from experimental data for vapor pressures, liquid densities and enthalpies of vaporization $\left(E^{d m}=52.5 \mathrm{~kJ} \mathrm{~mol}^{-1}\right.$ and $S^{d m}=46.2 \mathrm{~J} \mathrm{~mol}^{-1} \mathrm{~K}^{-1}$ for all acids).

\begin{tabular}{|l|l|l|l|l|l|l|l|l|}
\hline Fluid & $\begin{array}{l}\text { Temperature } \\
\text { range }(\mathrm{K})\end{array}$ & $\begin{array}{l}\varepsilon_{h}^{*} \\
\left(\mathrm{Jmol}^{-1}\right)\end{array}$ & $\begin{array}{l}\varepsilon_{s}^{*} \\
\left(\mathrm{Jmol}^{-1} \mathrm{~K}^{-1}\right)\end{array}$ & $\begin{array}{l}v_{s p, 0}^{*} \\
\left(\mathrm{~cm}^{3} \mathrm{~g}^{-1}\right)\end{array}$ & $s$ & $\begin{array}{l}\% \text { AAD } \\
\text { in } P^{s a t}\end{array}$ & $\begin{array}{l}\% \text { AAD } \\
\text { in } \rho^{\text {liq }}\end{array}$ & $\begin{array}{l}\text { AAD } \\
\text { in } H^{\text {vap }}\end{array}$ \\
\hline Acetic acid & $290-538$ & 4527.5 & 1.5654 & 0.8494 & 0.941 & 1.5 & 0.5 & 1.2 \\
\hline Propanoic acid & $287-542$ & 4431.7 & 1.3577 & 0.8919 & 0.908 & 0.4 & 1.0 & 2.4 \\
\hline Butanoic acid & $286-536$ & 4394.2 & 1.6826 & 0.9075 & 0.888 & 1.6 & 1.5 & 2.0 \\
\hline
\end{tabular}

$1 \% A A D=(100 / n) \sum_{i}\left|\left(X_{i}^{c a l}-X_{i}^{\exp }\right) / X_{i}^{\exp }\right|$, where $\mathrm{X}$ stands for the property of interest (vapor pressure, $P^{\text {sat }}$, saturated liquid density, $\rho^{\text {liq }}$, and enthalpy of vaporization, $H^{\text {vap }}$ ) and $n$ is the number of experimental data. 


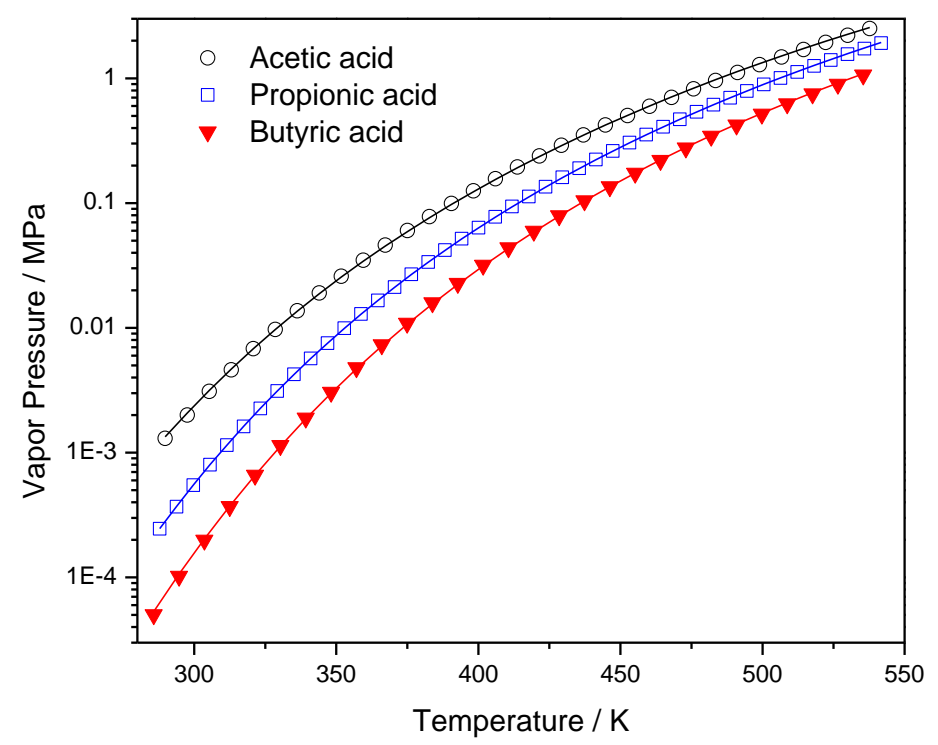

Figure 1. Vapor pressures of acetic, propionic and butyric acid. DIPPR ${ }^{42}$ correlations (symbols) and NRHB calculations (lines).

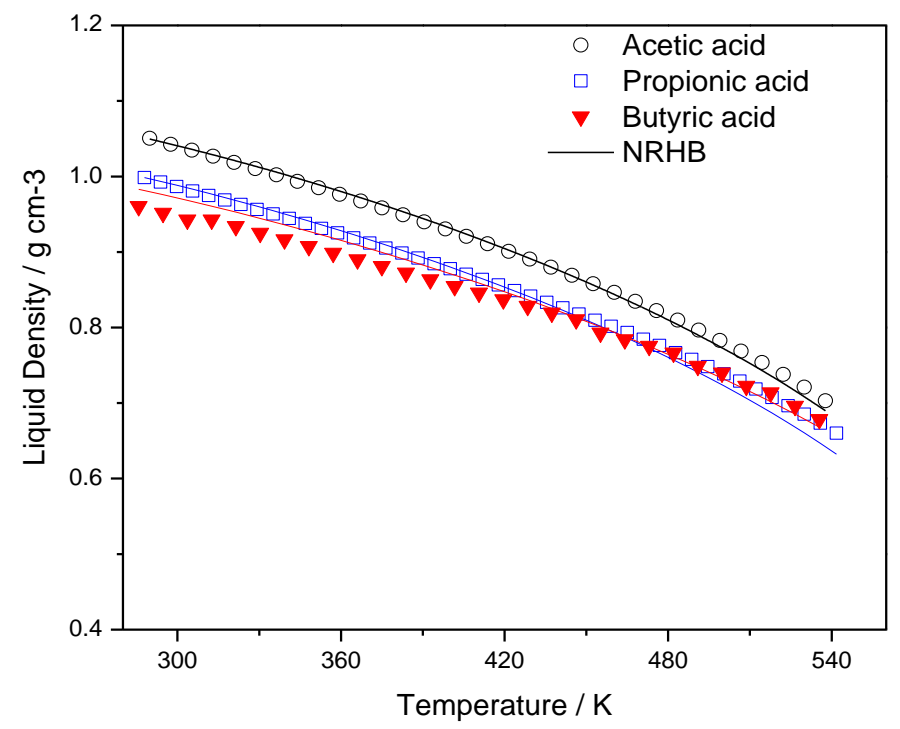

Figure 2. Saturated liquid densities of acetic, propionic and butyric acid. DIPPR ${ }^{42}$ correlations (symbols) and NRHB calculations (lines). 


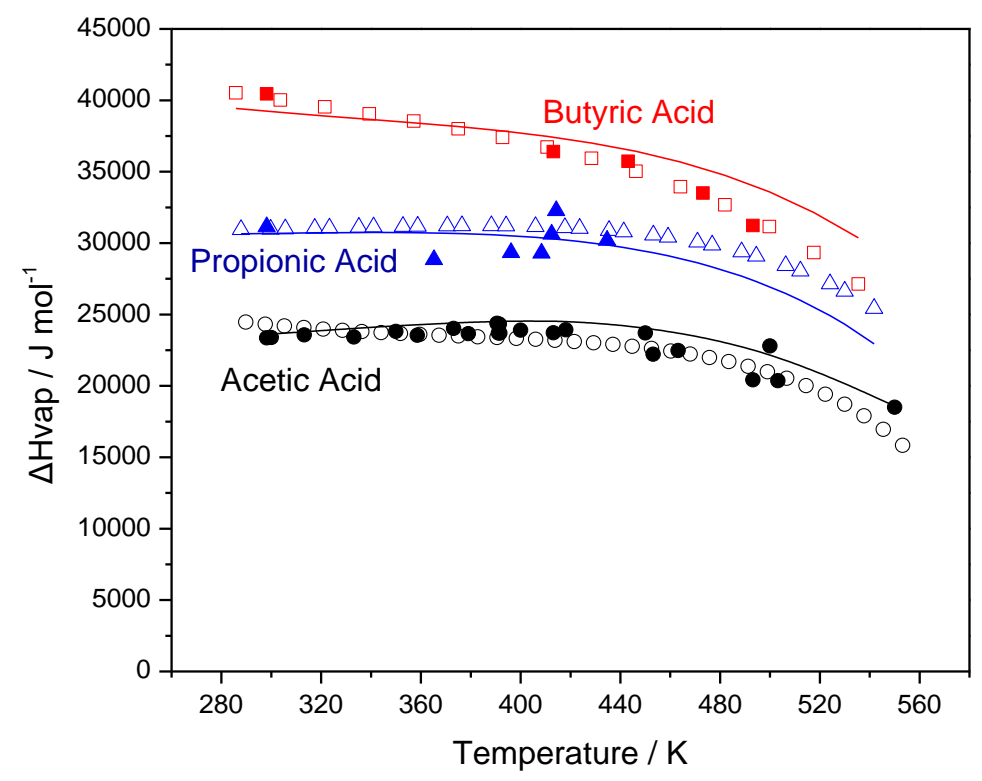

Figure 3. Enthalpies of vaporization for acetic, propionic and butyric acid. DIPPR ${ }^{42}$ correlations (open symbols), experimental data ${ }^{42}$ (solid symbols) and NRHB calculations (lines).

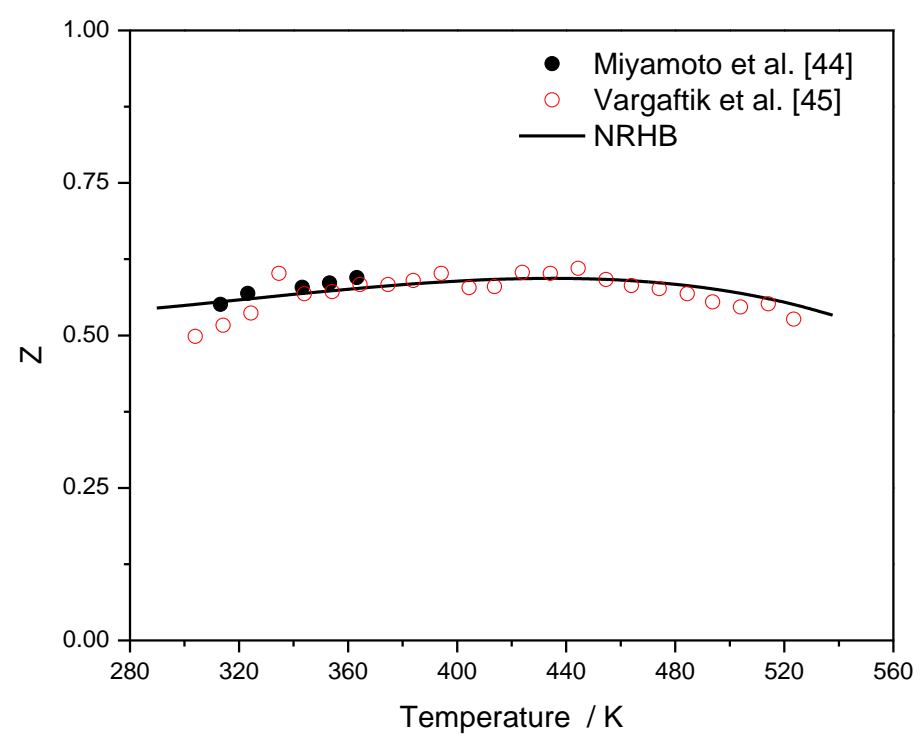

Figure 4. Acetic acid compressibility factors for saturated vapor. Experimental data (symbols) ${ }^{44,45}$ and NRHB calculations (line). 
As presented in Figures 1 and 2, the NRHB model satisfactorily describes the vapor pressure and saturated liquid densities. However, as already mentioned, the dimerization in the vapor phase strongly affects properties such as the vapor densities (or equivalently the vapor phase compressibility factors) and the heats of vaporization, which are satisfactorily described by the NRHB model, as shown in Figures 3 and 4. The model presents a maximum in the compressibility factors curve (Figure 4), which is also shown by the experimental data. Here it is worth mentioning that such low molecular weight acids present a large deviation form ideal gas behavior ( $Z$ significantly deviates from unity) due to strong dimerization even at low pressures and even below $1 \mathrm{~atm}^{3}$

It is worth mentioning that the heat of vaporization and the compressibility factors are not satisfactorily described if the formation of dimers is not accounted for, in accordance with previous literature studies. ${ }^{3,29}$ This is clearly shown in Figure 5, where the NRHB calculations are compared with the literature data for two cases: modeling acetic acid assuming a hydrogen bonding behavior similar to that of alcohols (one proton donor and one proton acceptor on every molecule that are able to cross associate and form oligomers), which is a frequently used approach ${ }^{25}$, and restricting the formation of hydrogen bonds to the formation of cyclic dimers. When the formation of linear oligomers is assumed, the model cannot satisfactorily describe the enthalpies of vaporization, in agreement with a previous study ${ }^{29}$. In that case, the model predictions are closer to the enthalpies of vaporization of 2-propanol, which can be considered as an associating homomorph of acetic acid, which does not form cyclic dimers. Consequently, the difference in the calculated heat of vaporization between the two modeling approaches is mainly attributed to the existence of dimerized molecules in the vapor phase.

As shown in Figure 6, the dimerization is significant even at low pressures or high temperatures. From this figure it is clear that the model predicts a non-negligible fraction of dimerized molecules in the vapor phase in agreement with the trend shown by the literature data ${ }^{46}$ and other models, such as the association scheme proposed by Janecek and Paricaud $^{29}$, who mention that the part of molecules in non-bonded state and the fraction of molecules bonded in cyclic dimers is higher in the vapor phase. It is worth mentioning here that the used literature data ${ }^{46}$ were obtained assuming only monomers and dimers in the vapor phase, which, seems to be a reasonable approximation ${ }^{61-63}$. 
At the same time, as also shown in Figure 6, the model predicts a liquid phase (the part of the curve at low temperatures in Figure 6) that mostly contains associated molecules. According to the model of Janecek and Paricaud ${ }^{29}$, chain oligomers dominate in the liquid state of acetic acid. Kamath et al. ${ }^{5}$ have conducted molecular simulation studies and suggest that hydrogen bonding between hydroxyl groups is possible, but it occurs with much less frequency than between hydroxyl and carbonyl groups. Also, neutron scattering experimental data obtained from the same group show that there are exactly four atoms within a radius of $2.12 \AA$ from the hydroxyl hydrogen, which suggests a planar dimer structure $^{5}$. Nevertheless, at this point more detailed experimental data are needed in order to achieve a more physically correct representation of the liquid-phase structure.

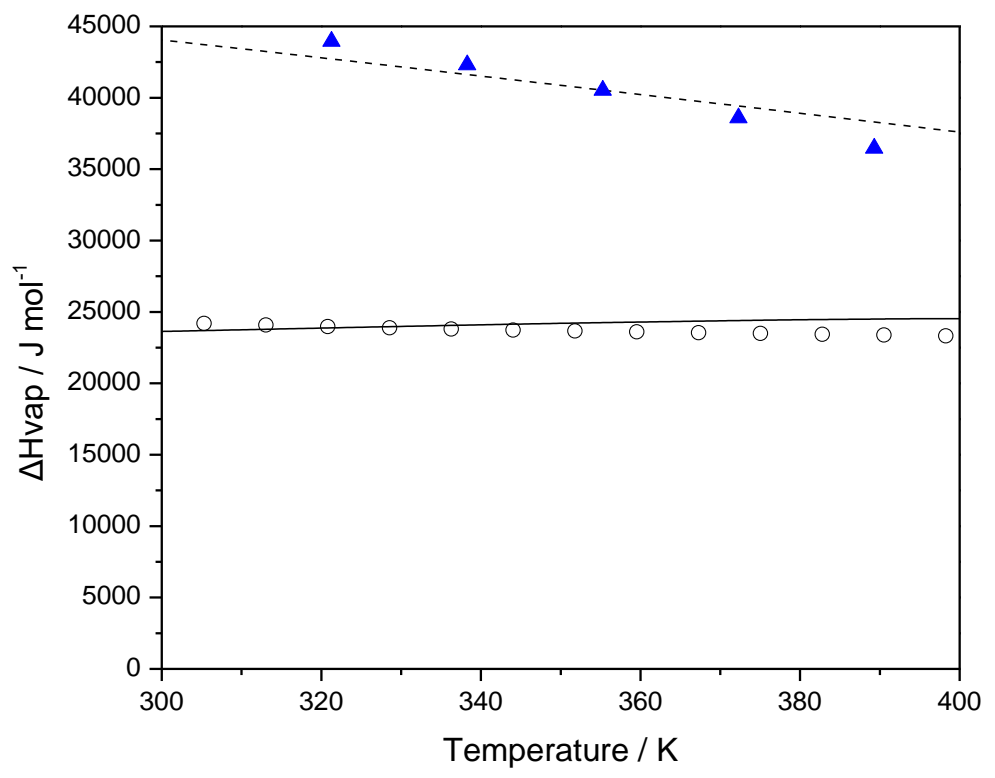

Figure 5. Enthalpy of vaporization for acetic acid. DIPPR ${ }^{42}$ correlations (open symbols) and NRHB calculations (lines) using two modeling approaches: assuming the formation of cyclic dimers (solid line) and the formation of linear oligomers (dash line). The DIPPR ${ }^{42}$ correlations for the enthalpy of vaporization of 2-propanol is also shown (solid symbols). 


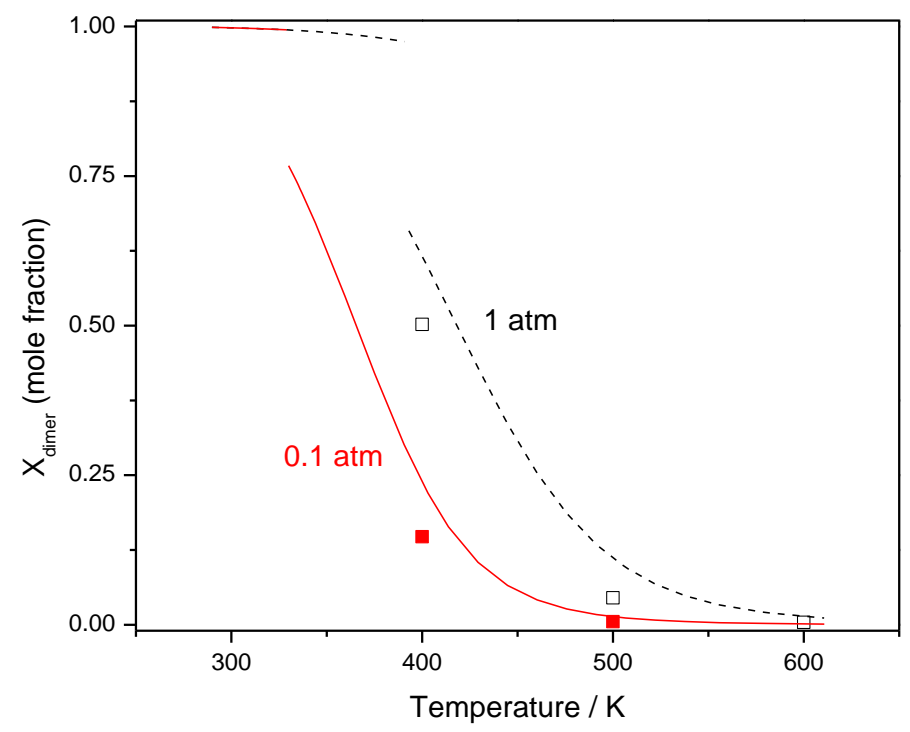

Figure 6. Fraction of dimers for acetic acid at low pressures. Literature data ${ }^{46}$ (points) and NRHB calculations (lines).

\subsection{Binary Mixtures}

Having the NRHB parameters for pure fluids, the model can be used to predict thermodynamic properties of mixtures, including phase equilibria and thermal properties. As already mentioned, the accurate prediction of thermal properties, which are usually not investigated when testing thermodynamic models, is the recommended way in order to check if the various intermolecular interactions are properly accounted for and, as it will be revealed next in this section, allows the drawing of interesting conclusions for the interplay between various types of molecular interactions, which are not easily revealed by experimental measurements. The latter is feasible since the NRHB model permits the calculation of separate contributions to the enthalpies of mixing, namely, from hydrogen bonding and physical (non-hydrogen bonding: dispersion and polar) interactions.

Next, the NRHB model will be used to describe the enthalpies of mixing for binary systems of organic acids and inert solvents and in mixtures of two carboxylic acids using the acids' parameters obtained in this study and parameters for the rest of the fluids from literature. $^{21,22,32,33}$ All correlations shown in Figures 7-14 were performed using one temperature independent binary interaction parameter, $k_{i j}$, which is presented in Table 2. However, as presented in Figures 7-9, most often such experimental data are in fair 
agreement with each other and sometimes the temperature dependence of the reported excess enthalpies is not easily revealed.

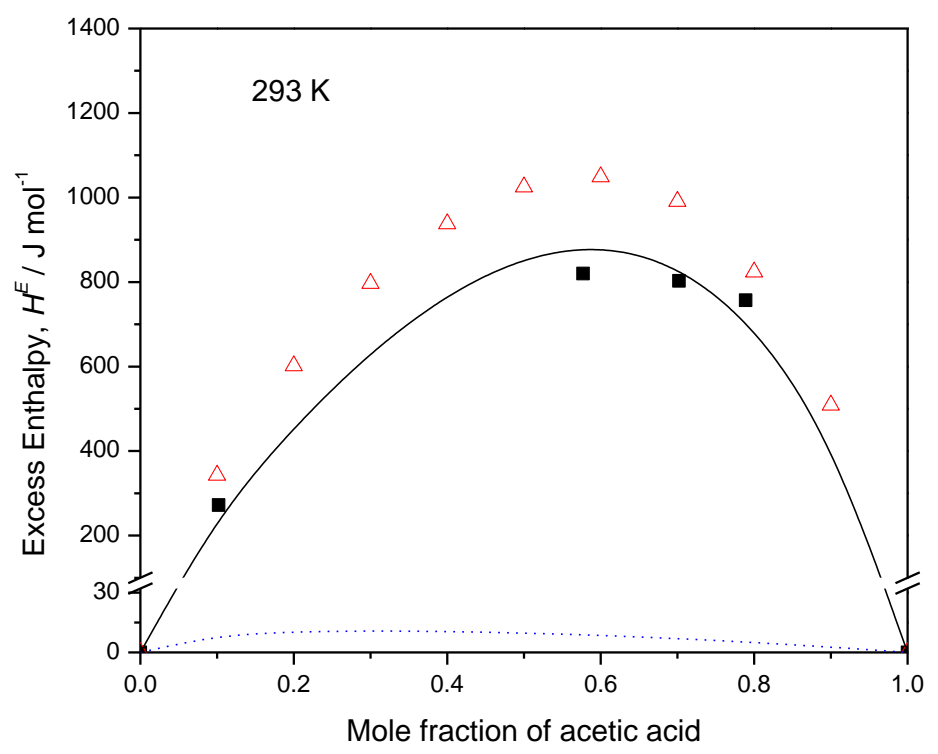

Figure 7. Excess enthalpies for the acetic $-n$-heptane mixture. Experimental data ${ }^{47,48}$ (points) and NRHB calculations (lines). The solid lines correspond to the total property, while the dotted lines to the hydrogen bonding contribution.

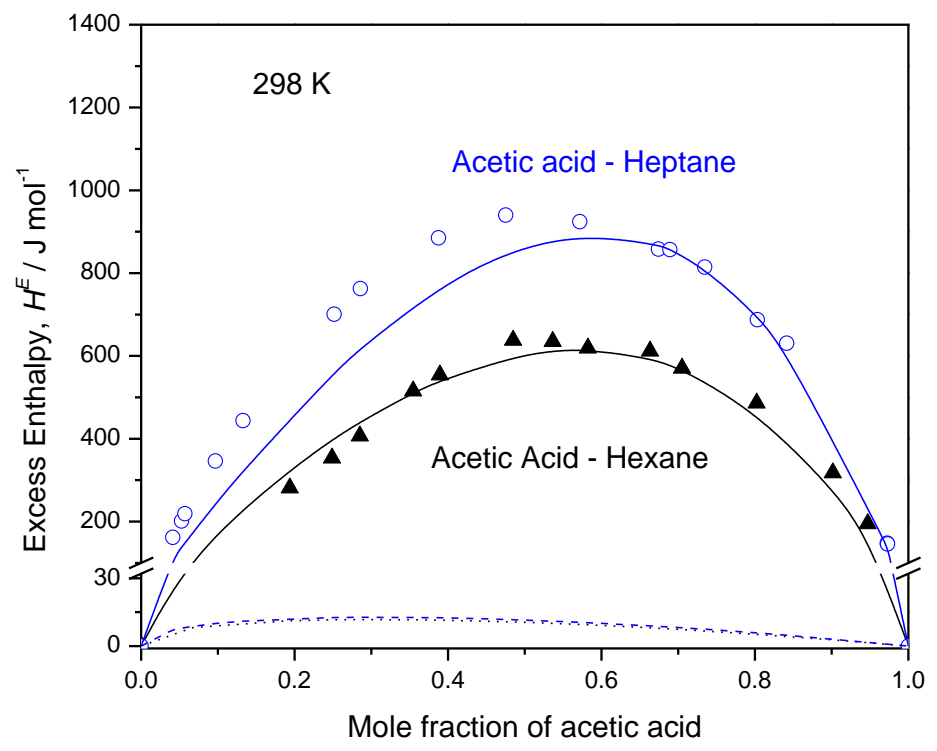

Figure 8. Excess enthalpies for acetic acid $-n$-hexane and acetic acid $-n$-heptane mixtures. Experimental data ${ }^{49}$ (points) and NRHB calculations (lines). The solid lines correspond to the total property, while the dotted lines to the hydrogen bonding contribution. 

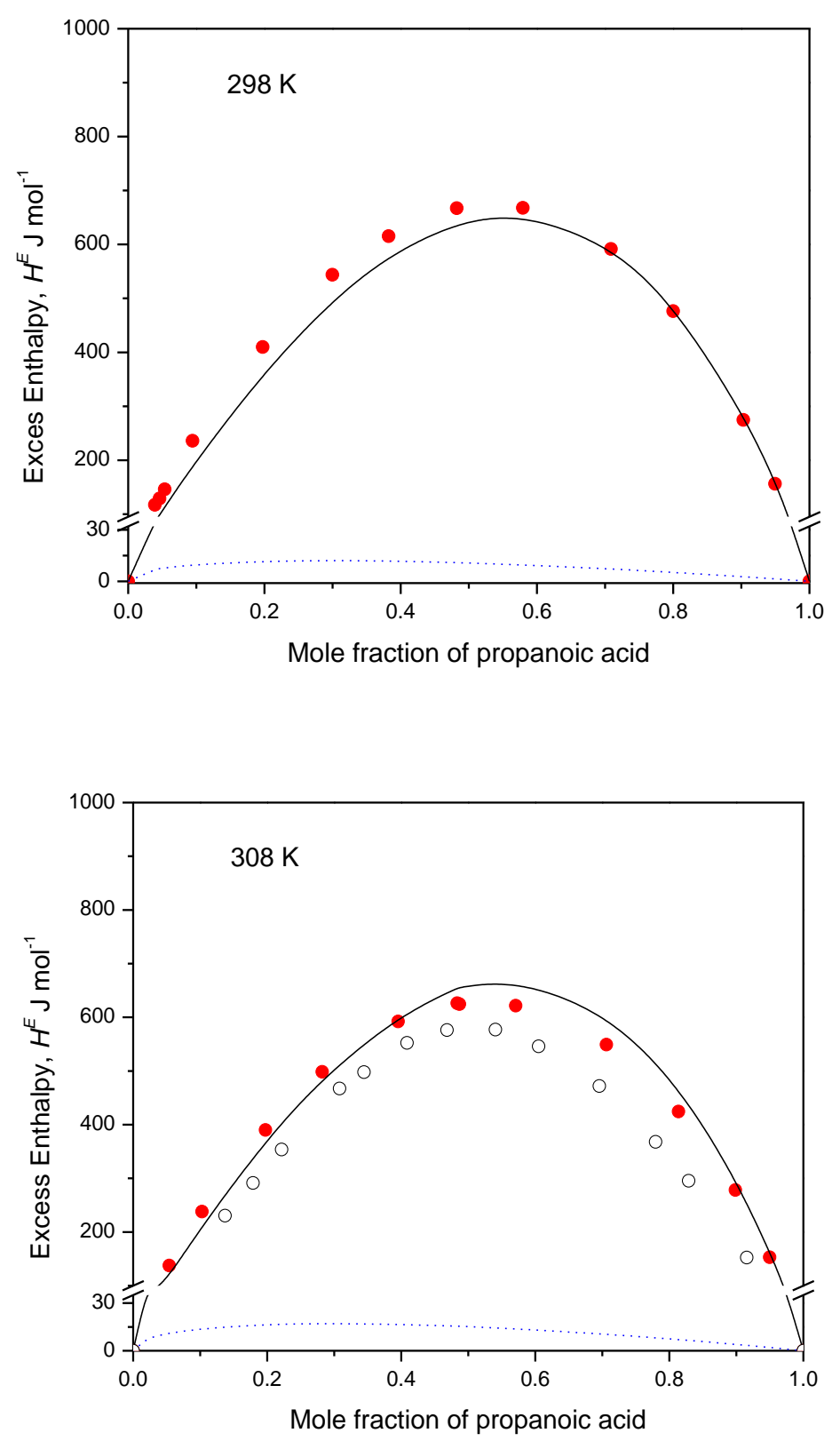

Figure 9. Excess enthalpies for the propanoic acid $-n$-heptane system. Experimental data $^{50,51}$ (points) and NRHB calculations (lines). The solid lines correspond to the total property, while the dotted lines to the hydrogen bonding contribution. 


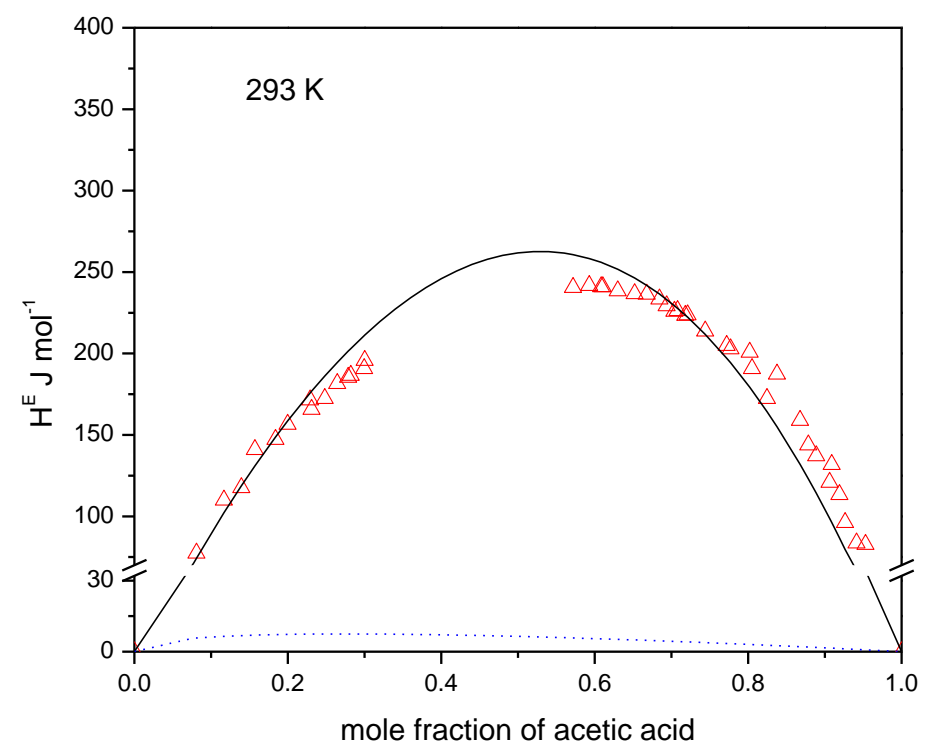

Figure 10. Excess enthalpies for the acetic acid - carbon tetrachloride system. Experimental data ${ }^{52,53}$ (points) and NRHB calculations (lines). The solid lines correspond to the total property, while the dotted lines to the hydrogen bonding contribution.

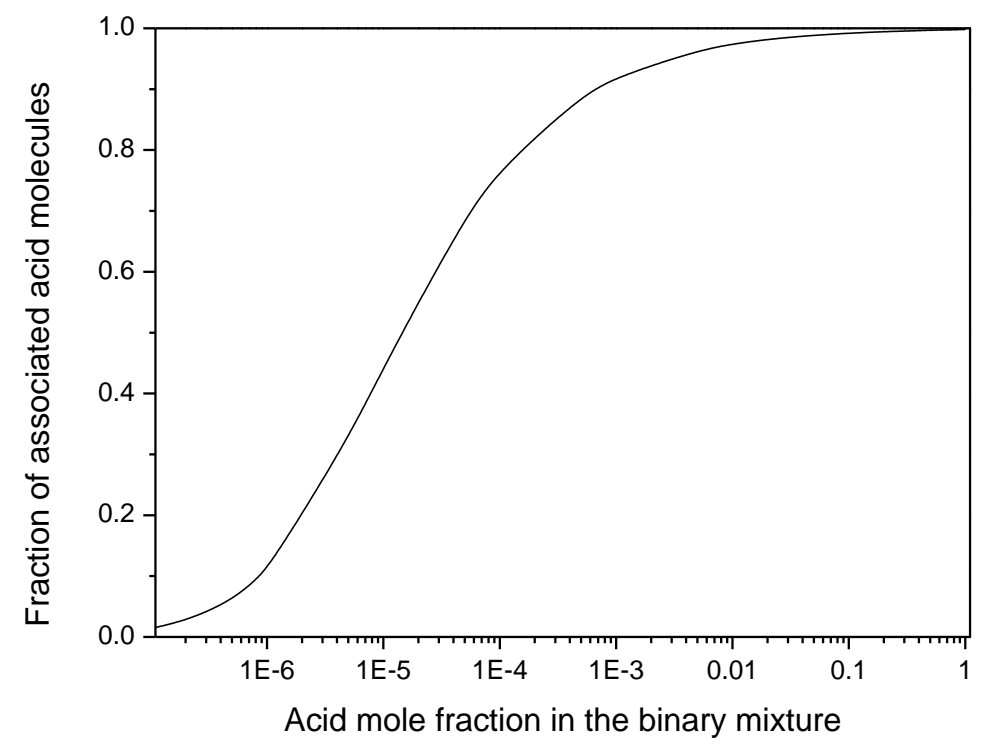

Figure 11. Fraction of associated acid molecules in the propanoic acid $-n$-heptane binary mixture at $313 \mathrm{~K}$. 
Nevertheless, the available experimental data for the heats of mixing of low molecular weight carboxylic acids with hydrocarbons show that the mixing is an endothermic process. Such behavior is rather expected and is usually attributed to the hindering of hydrogen bonding and the weakening of dipole - dipole interactions between acid molecules as more hydrocarbon molecules are added to the system. However, one cannot easily tell to which extent the various contributions from intermolecular forces affect the measurable property (heat of mixing). To answer such question, the NRHB model was applied to predict the separate contributions, from hydrogen bonding and from physical (polar and dispersive) intermolecular interactions, to the total values of mixing enthalpies. As shown in Figures $7-10$, the model predicts that the hydrogen bonding contribution is positive (endothermic), which means that some hydrogen bonds break upon mixing with inert hydrocarbon molecules, but such contribution is very small compared to the contribution from physical interactions. In other words, the acid molecules are strongly associated in the liquid state and the number of the hydrogen bonded molecules is not significantly altered by the addition of an inert solvent. This is clearly shown in Figure 11, where the predictions of the model for the fraction of associated acid molecules versus the mole fraction of the acid in the binary mixture is presented for propanoic acid $-n$-heptane system. Furthermore, as shown in Figure 12, where the hydrogen bonding contribution to the total excess enthalpy ratio for propanoic acid $-n$-heptane mixture is presented, the contribution of hydrogen bonding becomes significant only in very dilute mixtures.

As shown in Figure 8, where the excess enthalpies of the acetic acid $-n$-hexane and acetic acid $-n$-heptane systems are compared, higher values are observed for the mixture with $n$-heptane. As the size of the hydrocarbon molecule increases, the hindering of the physical interactions (dispersive and polar) between acid molecules becomes more pronounced. This behavior is well described by the NRHB model. 


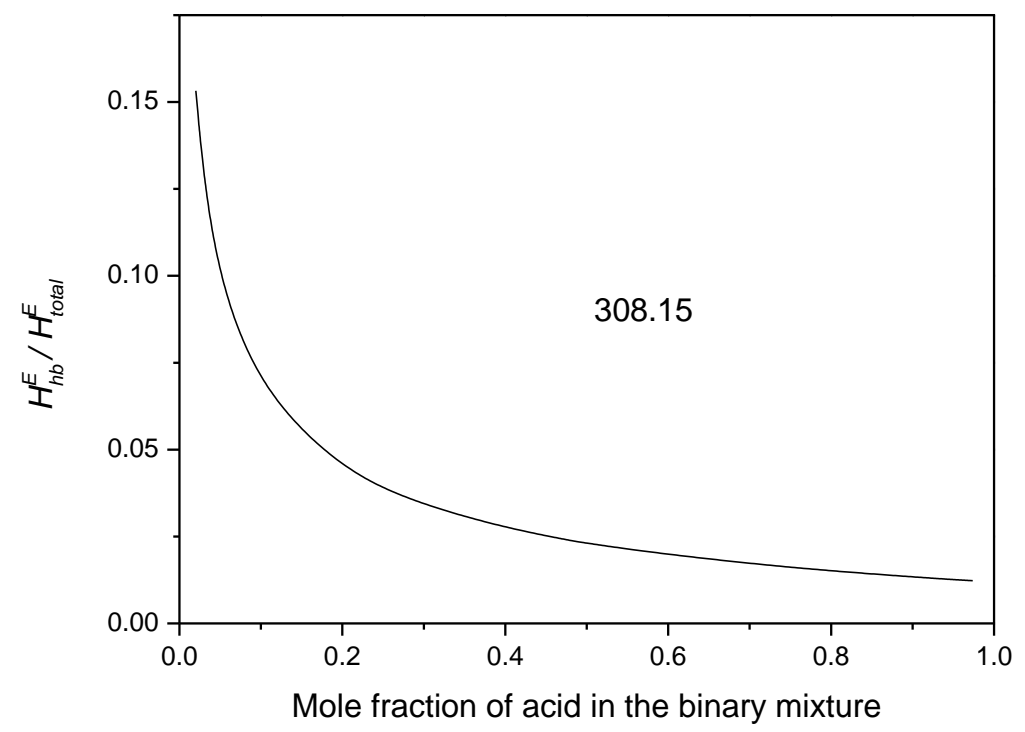

Figure 12. The ratio of the hydrogen bonding component to the total excess enthalpy for propanoic acid $-n$-heptane mixture at $308.15 \mathrm{~K}$.

Next, the model was applied to describe the excess enthalpies of binary mixtures that contain two carboxylic acids. In all cases the hydrogen bonding parameters for the cross association interactions were considered equal to those for self association interactions. The results, which are compared with the limited experimental data that are available in the literature, are shown in Figures 13 and 14. From such figures it can be seen that in systems of two organic acids, the heats of mixing are positive, which means that the mixing is an endothermic process. According to the NRHB model, in mixtures of two acids, the hydrogen bonding contribution to the total excess enthalpy is very small (dotted lines in Figure 13). This behavior is rather expected, since, as already mentioned above, the dimerization enthalpy is rather constant for low molecular weight organic acids. Consequently, the addition of another acid results in a decrease of the self-association interactions, but, at the same time, more cross-association interactions occur in the system. In other words, upon addition of another acid, dimers of molecules of the same kind break but dimers consisting of molecules of different kind appear in the system. As shown by the calculations, the net effect of such rearrangement of hydrogen bonds is an insignificant contribution to the total enthalpies of mixing. This conclusion is further supported by the 
vapor-liquid equilibrium (VLE) behavior of such binary mixtures, which present relatively strong hydrogen bonding behavior, but, at the same time, present a VLE phase diagram that resembles the behavior of ideal systems (Figure 15). As already known, the appearance of such VLE phase diagram, alone, indicates that the cross- intermolecular interactions are almost as strong as the interactions between molecules of the same kind.

However, the comparison of Figures 13 and 8 reveals another interesting phenomenon. The enthalpies of mixing in systems with two acids are almost one order of magnitude lower than the corresponding ones for mixtures of acids with hydrocarbons ( $n$-hexane can be considered as a non-associating and non-polar homomorph of butanoic acid). Having in mind the small contribution of dimerization to the heats of mixing, the lower values that are observed can be attributed to the acid dipole-dipole interactions. Such intermolecular forces are significantly hindered in mixtures of acids with hydrocarbons, as the molecules of the non-polar inert solvent are placed between acid species (monomers and dimers).

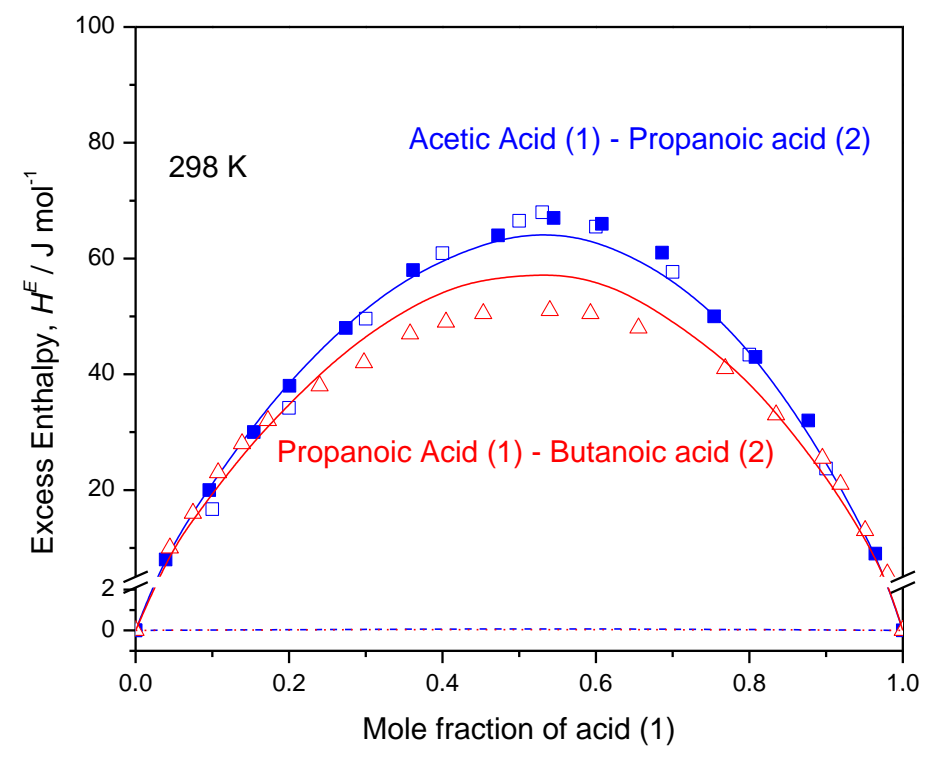

Figure 13. Excess enthalpies for the mixtures of acetic acid - propanoic acid and for propanoic acid - butanoic acid systems. Experimental data ${ }^{54}$ (points) and NRHB calculations (lines). The solid lines correspond to the total property, while the dotted lines to the hydrogen bonding contribution. 

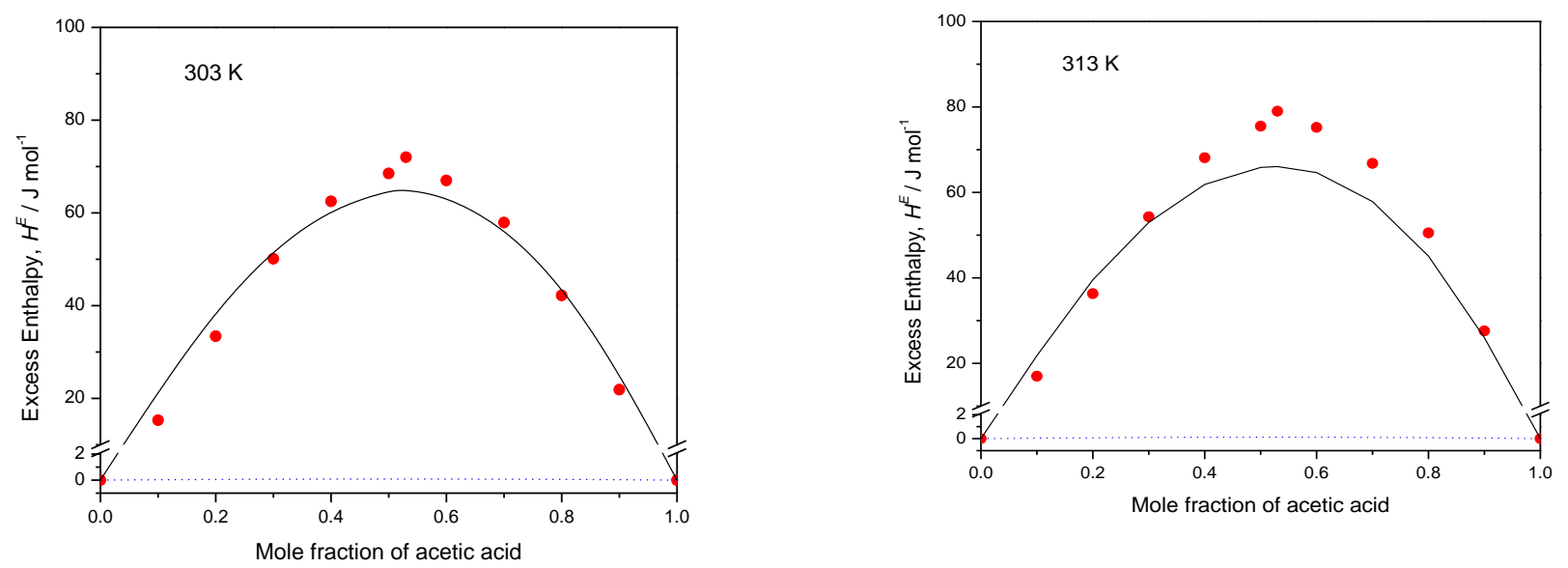

Figure 14. Excess enthalpies for the acetic acid - propanoic acid mixture. Experimental data (points) ${ }^{55}$ and NRHB calculations (lines). The solid lines correspond to the total property, while the dotted lines to the hydrogen bonding contribution.

As mentioned above, all calculations of the excess enthalpies were performed using one temperature independent binary interaction parameter, $k_{i j}$ (Table 2), which was optimized by the available $H^{E}$ experimental data. It was observed that the calculated values of the excess enthalpy were very sensitive to small changes of such binary parameter. Usually, when testing thermodynamic models, the binary parameters are adjusted solely to phase equilibrium data, neglecting the thermal properties. It is then often observed that the model fail to satisfactorily describe the enthalpies of mixing, although the phase behavior, and especially the VLE, is accurately correlated. Such binary model parameter reflects a correction for the strength of cross- intermolecular physical (non-hydrogen bonding) interactions and, consequently, the calorimetric data are, probably, the most appropriate data for its optimization. Optimization of the binary parameters solely to phase equilibrium and especially VLE data may result in accurate correlations for this property, but may effectively hide limitations of the model. 


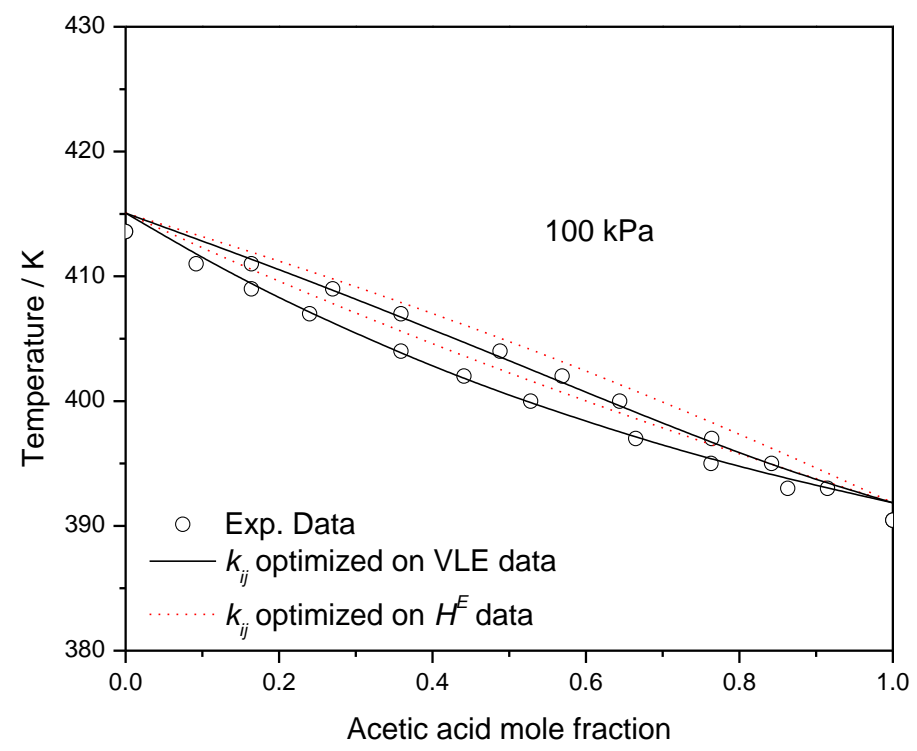

Figure 15. VLE for acetic acid - propanoic acid. Experimental data ${ }^{56}$ (points) and NRHB calculations (lines).

Consequently, in order to evaluate if the calculated binary parameters, which were optimized to $H^{E}$ data, are appropriate also for VLE calculations, the vapor liquid equilibrium was estimated for systems for which data are available, All the estimated binary parameters are shown in Table 2 and representative results are presented in Figures 15 and 16. It was observed that the values of the binary interaction parameters obtained from VLE data are close to the values that were estimated using only excess enthalpy data. As shown in Figures 15 and 16, the binary interaction parameters that were optimized using solely excess enthalpy data give a rather satisfactory description of the vapor - liquid equilibrium, although such calculations do not describe the experimental data as accurately as the parameters optimized by the VLE data. 
Table 2. Binary interaction parameters used for the calculations in acid mixtures

\begin{tabular}{|l|c|c|}
\hline System & $\begin{array}{c}1-k_{i j} \\
\text { (optimized using } H^{E} \text { data) }\end{array}$ & $\begin{array}{c}1-k_{i j} \\
\text { (optimized using VLE data) }\end{array}$ \\
\hline Acetic acid $-n$-hexane & 0.967 & 0.990 \\
\hline Acetic acid $-n$-heptane & 0.954 & 0.939 \\
\hline Acetic Acid - carbon tetrachloride & 0.986 & 0.967 \\
\hline Acetic acid - propanoic acid & 0.995 & 0.985 \\
\hline Propanoic acid $-n$-heptane & 0.970 & 0.965 \\
\hline Propanoic acid - butanoic acid & 0.997 & 0.982 \\
\hline
\end{tabular}

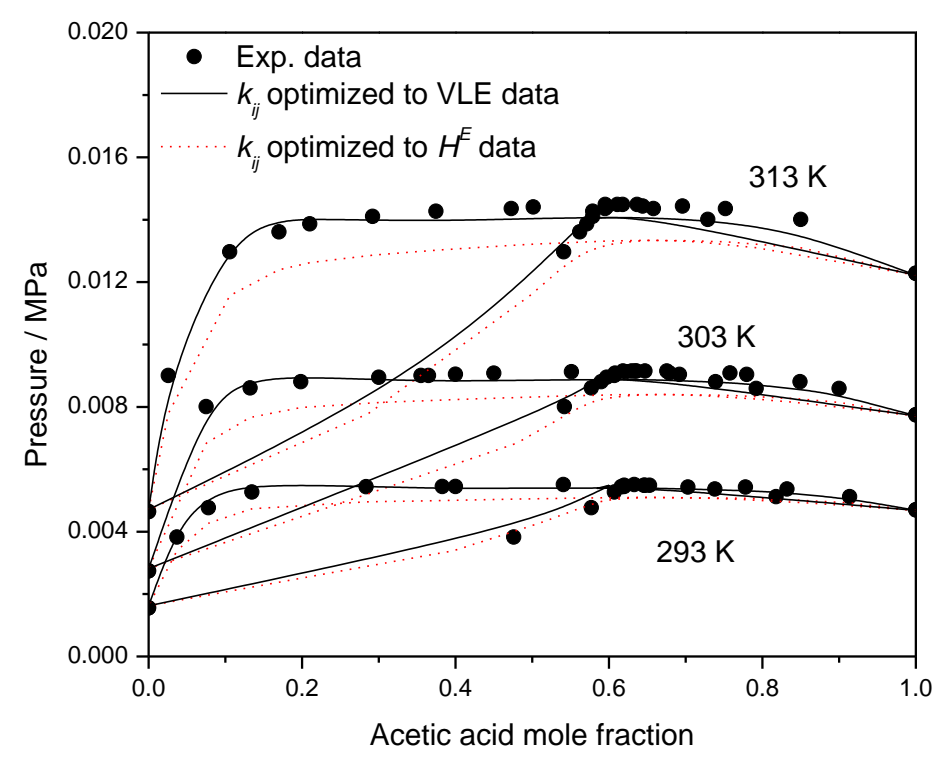



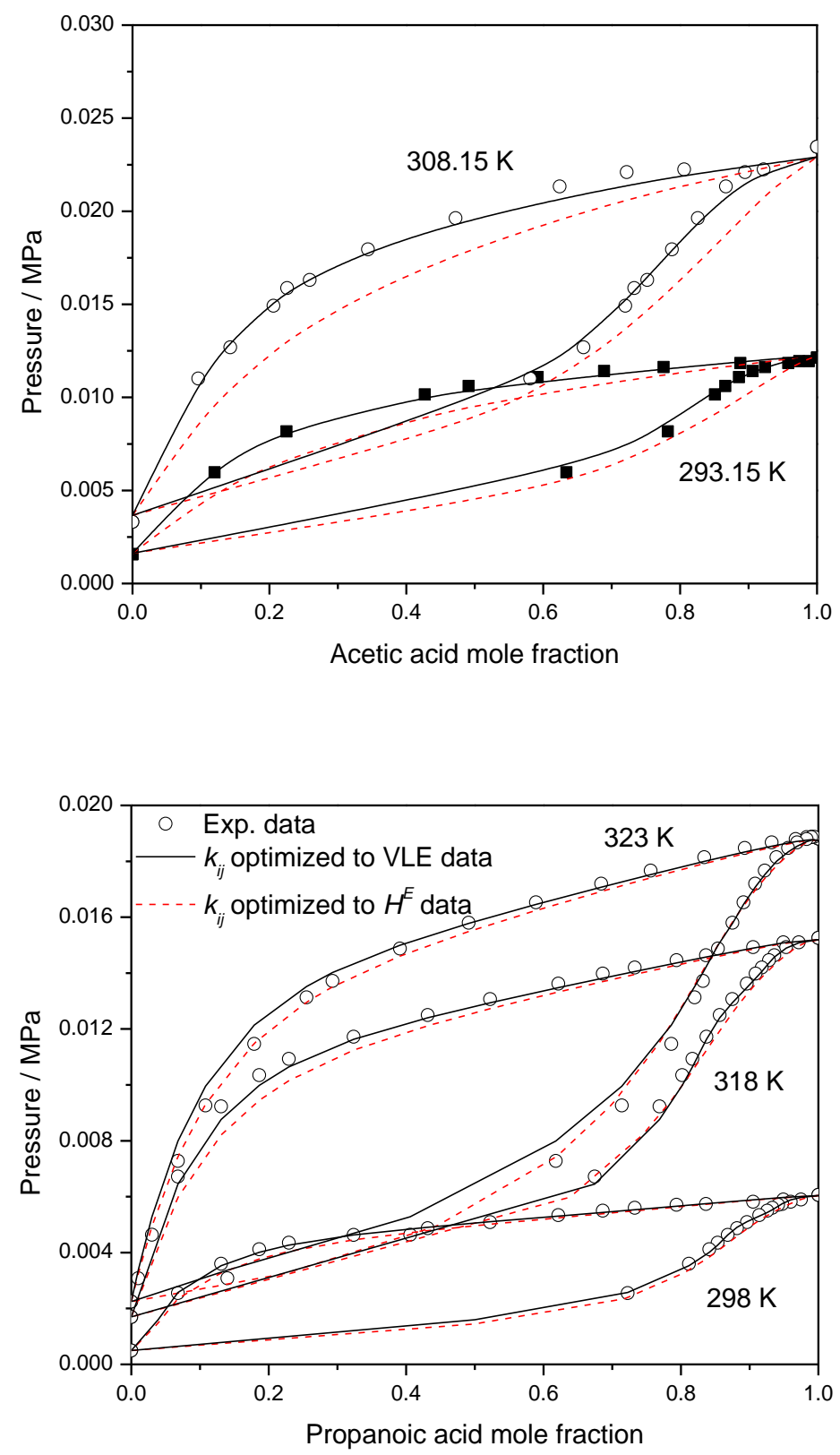

Figure 16. VLE for acetic acid - $n$-heptane (a) and acetic acid - carbon tetrachloride (b), propionic acid $-n$-heptane. Experimental data ${ }^{51,57-60}$ (points) and NRHB calculations (lines).

\section{Conclusions}

The association term of the Non-Random Hydrogen-Bonding theory was extended to describe systems with dimerized acid molecules and the model was applied to calculate the thermal 
properties, which are usually not considered when evaluating thermodynamic models and the vapor-liquid equilibrium of relevant fluid mixtures. The model permits the calculation of the separate contributions to the mixing enthalpies, namely, from hydrogen bonding and physical (non hydrogen bonding) interactions, while such "deconvolution" of the total property values permits the drawing of interesting conclusions. According to the NRHB model, in mixtures of organic acids with hydrocarbons (inert solvents), the contribution of hydrogen bonding is not significant, since the acid molecules are very strongly associated. Consequently, the endothermic mixing is mainly attributed to the hindering of dispersive and polar interactions between acid molecules upon the addition of inert solvent molecules. Furthermore, in systems with two carboxylic acids the rearrangement of hydrogen bonds due to the formation of cross associating dimers results in an insignificant contribution to the heats of mixing, since the dimerization enthalpy remains rather constant for low molecular weight carboxylic acids, as shown by the relevant experimental data.

\section{References}

[1] Tsivintzelis, I.; Kontogeorgis, G.M. Capabilities and Limitations of an Association Theory for Chemicals in Liquid or Supercritical Solvents. Ind. Eng. Chem. Res. 2012, 51, 13496-13517.

[2] Tsivintzelis, I.; Kontogeorgis, G.M. On the Predictive Capabilities of CPA for Applications in the Chemical Industry: Multicomponent Mixtures Containing Methyl-Methacrylate, Dimethyl-Ether or Acetic Acid. Chem. Eng. Res. Design, 2014, 92, 2947-2969.

[3] Vahid, A.; Elliott, J. R. Transferable Intermolecular Potentials for Carboxylic Acids and Their Phase Behavior. AIChE J. 2010, 56, 485-505.

[4] Pimentel, G. C.; McClellan, A.L. The Hydrogen Bond; W.H. Freeman and Company: New York and London, 1960.

[5] Kamath, G.; Cao, F.; Potoff, J.J. An Improved Force Field for the Prediction of the VaporLiquid Equilibria for Carboxylic Acids. J. Phys. Chem. B 2004, 108, 14130-14136. 
[6] Sanchez, I.; Lacombe, R.H. Statistical Thermodynamics of Polymer Solutions.

Macromolecules 1978, 11, 1145-1156.

[7] Sanchez, I.C.; Panayiotou C. Equations of State Thermodynamics of Polymer and Related Solutions, in Models for Thermodynamic and Phase Equilibria Calculations; Sandler, S., Ed.; Dekker: New York, 1994.

[8] Panayiotou, C.; Vera, J.H. Statistical Thermodynamics of $r$-mer Fluids and their Mixtures. Polymer J. 1982, 14, 681-694.

[9] Panayiotou, C.; Sanchez I.C. Hydrogen Bonding in Fluids: An Equation of State Approach. J. Phys. Chem. 1991, 95, 10090-10097.

[10] Panayiotou, C. The QCHB Model of Fluids and their Mixtures. J. Chem. Thermodyn. 2003, $35,349-381$.

[11] Panayiotou, C.; Stefanis E.; Tsivintzelis I.; Pantoula M.; Economou I. Nonrandom Hydrogen-Bonding Model of Fluids and their Mixtures 1. Pure Fluids. Ind. Eng. Chem. Res. 2004, 43, 6952-6606.

[12] Panayiotou, C.; Tsivintzelis, I.; Economou I.G. Nonrandom Hydrogen-Bonding Model of Fluids and their Mixtures. 2. Multicomponent Mixtures. Ind. Eng. Chem. Res. 2007, 46, 26282636.

[13] Chapman, W.G.; Gubbins, K. E.; Jackson, G.; Radosz, M. New Reference Equation of State for Associating Liquids. Ind. Eng. Chem. Res. 1990, 29, 1709-1721.

[14] Huang, H.S.; Radosz, M. Equation of State for Small, Large, Polydisperse and Associating Molecules. Ind. Eng. Chem. Res. 1990, 29, 2284-2294.

[15] Huang, H.S.; Radosz, M. Equation of State for Small, Large, Polydisperse and Associating Molecules: Extension to Fluid Mixtures. Ind. Eng. Chem. Res. 1991, 30, 1994-2005.

[16] Wertheim, M.S. Fluids with Highly Directional Attractive Forces. 1. Statistical Thermodynamics. J. Stat. Phys. 1984, 35, 19-34.

[17] Wertheim, M.S. Fluids with Highly Directional Attractive Forces. 2. Thermodynamic Perturbation-Theory and Integral-Equations. J. Stat. Phys. 1984, 35, 35-47.

[18] Wertheim, M.S. Fluids with Highly Directional Attractive Forces. 3. Multiple Attraction Sites. J. Stat. Phys. 1986, 42, 459-476. 
[19] Wertheim, M.S. Fluids with Highly Directional Attractive Forces. 4. Equilibrium Polymerization. J. Stat. Phys. 1986, 42, 477-492.

[20] Tsivintzelis, I.; Spyriouni, T.; Economou, I.G. Modeling of Fluid Phase Equilibria with Two Thermodynamic Theories: Non-Random Hydrogen Bonding (NRHB) and Statistical Association Fluid Theory (SAFT). Fluid Phase Equilib. 2007, 253, 19-28.

[21] Grenner, A.; Tsivintzelis, I.; Kontogeorgis, G.M.; Economou, I.G.; Panayiotou, C. Evaluation of the Non-Random Hydrogen Bonding (NRHB) Theory and the Simplified Perturbed-Chain-Statistical Associating Fluid Theory (sPC-SAFT). 1. Vapor-Liquid Equilibria. Ind. Eng. Chem. Res. 2008, 47, 5636-5650.

[22] Tsivintzelis, I.; Grenner, A.; Economou, I.G.; Kontogeorgis. Evaluation of the Non-Random Hydrogen Bonding (NRHB) Theory and the Simplified Perturbed-Chain-Statistical Associating Fluid Theory (sPC-SAFT) 2. Liquid - Liquid Equilibria and Prediction of Monomer Fraction in Hydrogen Bonding Systems. Ind. Eng. Chem. Res. 2008, 47, 5651-5659.

[23] Muro-Suñé, N.; Kontogeorgis, G.M.; Von Solms, N.; Michelsen, M.L. Phase Equilibrium Modelling for Mixtures with Acetic Acid Using an Association Equation of State. Ind. Eng. Chem. Res. 2008, 47, 5660-5668.

[24] Breil, M.B.; Kontogeorgis, G.M.; Behrens, P.K.; Michelsen M.L. Modeling of the Thermodynamics of the Acetic Acid-Water Mixture Using the Cubic-Plus-Association Equation of State. Ind. Eng. Chem. Res. 2011, 50, 5795-5805.

[25] Derawi, S.O.; Zeuthen, J.; Michelsen, M.L.; Stenby, E.H.; Kontogeorgis, G.M. Application of the CPA Equation of State to Organic Acids. Fluid Phase Equilib. 2004, 225, 107-113.

[26] Gross, J.; Sadowski, G.; Application of the Perturbed-Chain SAFT Equation of State to Associating Systems. Ind. Eng. Chem. Res. 2002, 41, 5510-5515.

[27] Stefanis, E.; Tsivintzelis I.; Panayiotou C. The Partial Solubility Parameters: An Equation of State Approach. Fluid Phase Equilib. 2006, 240, 144-154.

[28] Sear, R.P.; Jackson, G. Thermodynamic Perturbation Theory for Association into Doubly Bonded Dimers. Mol. Phys. 1994, 82, 1033-1048.

[29] Janecek, J.; Paricaud, P. Influence of Cyclic Dimer Formation on the Phase Behavior of Crboxylic Acids. J. Phys. Chem. B, 2012, 116, 7874-7882.

[30] Janecek, J.; Paricaud, P. Influence of Cyclic Dimer Formation on the Phase Behavior of Crboxylic Acids. II. Cross-Associating Systems. J. Phys. Chem. B, 2013, 117, 9430-9438. 
[31] Missopolinou, D.; Tsivintzelis, I.; Panayiotou C. Excess Enthalpies of Binary Mixtures of 2Ethoxyethanol with four Hydrocarbons at 298.15, 308.15, and 318.15 K. An Experimental and Theoretical Study. Fluid Phase Equilib. 2006, 245, 89-101.

[32] Tsivintzelis, I.; Economou, I.G.; Kontogeorgis, G.M. Modeling The Solid-Liquid Equilibrium in Pharmaceutical-Solvent Mixtures: Systems with Complex Hydrogen Bonding Behavior. AIChE J. 2009, 55, 756-770.

[33] Tsivintzelis, I.; Economou, I.G.; Kontogeorgis, G.M. Modeling the Phase Behavior in Mixtures of Pharmaceuticals with Liquid or Supercritical Solvents. J. Phys. Chem. B, 2009, $113,6446-6458$.

[34] Tsivintzelis, I.; Kontogeorgis, G.M. Modeling the vapor-liquid equilibria of Polymer-Solvent Mixtures: Systems with Complex Hydrogen Bonding Behavior. Fluid Phase Equilib. 2009, 280, 100-109.

[35] Tsioptsias, C.; Tsivintzelis, I.; Panayiotou C. Equation-of-State Modeling of Mixtures with Ionic Liquids. Phys. Chem. Chem. Phys. 2010, 12, 4843-4851.

[36] Tsivintzelis, I.; Panayiotou, C. Molecular Thermodynamics of Hydrogen-Bonded Systems, in Handbook of Surface and Colloid Chemistry; Birdi, K.S., Ed.; Fourth edition, CRC Press: Boca Raton, London, New York, 2016.

[37] Missopolinou, D.; Panayiotou, C. Hydrogen-Bonding Cooperativity and Competing Interand Intramolecular Associations: A Unified Approach. J. Phys. Chem. A, 1998, 102, 35743581.

[38] Kontogeorgis, G.M.; Folas, G.K. Thermodynamic models for industrial applications. From Classical and advanced mixing rules to association theories; John Wiley and Sons: 2010.

[39] Tsivintzelis, I.; Ali, S.; Kontogeorgis G.M. Modeling Systems Relevant to the Biodiesel Production Using the CPA Equation of State. Fluid Phase Equilib. 2016, 430, 75-92.

[40] Clague, A.D.H.; Bernstein, H.J.; The Heat of Dimerization of Some Carboxylic Acids in the Vapour Phase Determined by a Spectroscopic Method. Spectrochimica Acta, 1969, 25A, 593596.

[41] Murthy, A.S.N.; Rao, C.N.R. Spectroscopic Studies of the Hydrogen Bond, Appl. Spectrosc. Rev. 1968, 2, 69-191.

[42] DIPPR 801 Thermophysical Property Database and DIADEM Predictive Proffesional, version 5.0.1, 2011. 
[43] Freeman, J.R.; Wilson, G.M.; High temperature PVT Properties of Acetic Acid/Water Mixtures. AIChE Symposium Series, 1985, 81, 1-13.

[44] Miyamoto, S.; Nakamura, S.; Iwai, Y.; Arai, Y. Measurement of Vapor-Phase Compressibility Factors of Monocarboxylic Acids Using a Flow-Type Apparatus and Their Association Constants. J. Chem. Eng. Data, 1999, 44, 48-51.

[45] Vargaftik, N.B.; Vinogradov, Y.K.; Yargin, V.S. Handbook of Physical Properties of Liquids and Gases: Pure Substances and Mixtures; 3rd edition, Begell House: New York, 1996.

[46] Chao, J.C.; Zwolinski, B.J. Ideal Gas Thermodynamic Properties of Methanoic and Ethanoic Acids. J. Phys. Chem. Ref. Data, 1978, 7, 363-377.

[47] Liszi, J. Acta. Chim. Acad. Sci. Hung. 1970, 66, 411-430, as reported in Christensen, J.J.;

Hanks, R.W.; Izatt R.M. Handbook of Heats of Mixing; J. Wiley and Sons: New York, Chichester, Brisbane, Toronto, Singapore, 1982.

[48] Woycicka, M. Untersuchungen über die Mischungswarmen in den Systemen: EssigsäurePyridin-n-Paraffine, Pure Appl. Chem. 1961, 2, 147-153.

[49] Nagata, I.; Nagashima, M.; Kazuma, K.; Nakagawa, M. Heats of Mixing for Binary Systems and Data Reduction Based on a Triplet Model of Guggenheim. J. Chem. Eng. Jpn. 1975, 8, 261-265.

[50] Geisler, G.; Suehnel, K.; Quitzsch, K. Z. Phys. Chem. (Leipzig) 1973, 254, 261-270, as reported in Christensen, J.J.; Hanks, R.W.; Izatt R.M. Handbook of Heats of Mixing; J. Wiley and Sons: New York, Chichester, Brisbane, Toronto, Singapore, 1982.

[51] Lark, B.S.; Banipal, T.S. Excess Enthalpies and Excess Volumes of some Carboxylic Acid Mixtures with n-Heptane. Thermochim. Acta. 1985, 91, 141,149

[52] Liszi, J. Magy. Kem. Foly. 1969, 75, 447-451, as reported in Christensen, J.J.; Hanks, R.W.; Izatt R.M. Handbook of Heats of Mixing; J. Wiley and Sons: New York, Chichester, Brisbane, Toronto, Singapore, 1982.

[53] Liszi, J. Acta Chim. Acad. Sci. Hung. 1970, 63, 371-383, as reported in Christensen, J.J.; Hanks, R.W.; Izatt R.M. Handbook of Heats of Mixing; J. Wiley and Sons: New York, Chichester, Brisbane, Toronto, Singapore, 1982. 
[54] Gallego, A.A. An. Quim. 1986, 82, 241, as reported in Christensen, J.J.; Hanks, R.W.; Izatt R.M. Handbook of Heats of Mixing; J. Wiley and Sons: New York, Chichester, Brisbane, Toronto, Singapore, 1982.

[55] Hasse, R.; Jansen, H.-J.; Winter, B. Z Naturforsch., 1983, 38a, 1400, as reported in Christensen, J.J.; Hanks, R.W.; Izatt R.M. Handbook of Heats of Mixing; J. Wiley and Sons: New York, Chichester, Brisbane, Toronto, Singapore, 1982.

[56] Kato, M.; Yoshikawa, H.; Yamaguchi, M. Vapor-Liquid Equilibrium Measurements of Three Binary Systems Made of Formic Acid, Acetic Acid and Propionic Acid by the DewBubble Point Temperature Method. Fluid Phase Equilib. 1990, 54, 47-56.

[57] Markuzin, N. P.; Pavlova, L. M., Zh. Prikl. Khim. (Leningrad), 1971, 44, 311, as reported in Vapor- Liquid Equilibria. Vol. 1, Hydrocarbons Part 1. Maczynski, A., Bok, A., Oracz, P., Skrzecz, A., Eds.; Thermodynamic Data Center: Warsav, 1997.

[58] Plekhotkin, V.F.; Markuzin N.P. Fiz. Khim. Svoistva Rastvorov Leningrad, Editor Rosanov 1964, 12.

[59] Tamir, A.; Dragoescu, C.; Apelblat, A.; Wisniak, J. Heats of Vaporization and Vapor-Liquid Equilibria in Associated Solutions Containing Formic Acid, Acetic Acid, Propionic Acid and Carbon Tetrachloride. Fluid Phase Equilib. 1983, 10, 9-42

[60] Schuberth, C.H. Vapor-Liquid-Equilibria for Propionic Acid + n-Heptane System at $50^{\circ} Z$. Phys. Chem. (Leipzig), 1981, 262, 945-952.

[61] Togeas, J.B. Acetic Acid Vapor: 2. A Statistical Mechanical Critique of Vapor Density Experiments. Phys. Chem. Chem. Phys. 2005, 109, 5438-5444.

[61] Chocholousova, J.; Vacek, J; Hobza P., Acetic Acid Dimer in the Gas Phase, Nonpolar Solvent, Microhydrated Environment, and Dilute and Concentrated Acetic Acid: Ab Initio Quantum Chemical and Molecular Dynamics Simulations. J. Phys. Chem. A 2003, 107, 30863092.

[62] Goubet, M.; Soulard, P.; Pirali, O.; Asselin, P.; Real, F.; Gruet, S.; Huet, T.R.; Roy, P.; Georges, R. Standard free energy of the equilibrium between the trans-monomer and the cyclic-dimer of acetic acid in the gas phase from infrared spectroscopy. Phys. Chem. Chem. Phys., 2015, 17, 7477-7488. 
TOC graphic
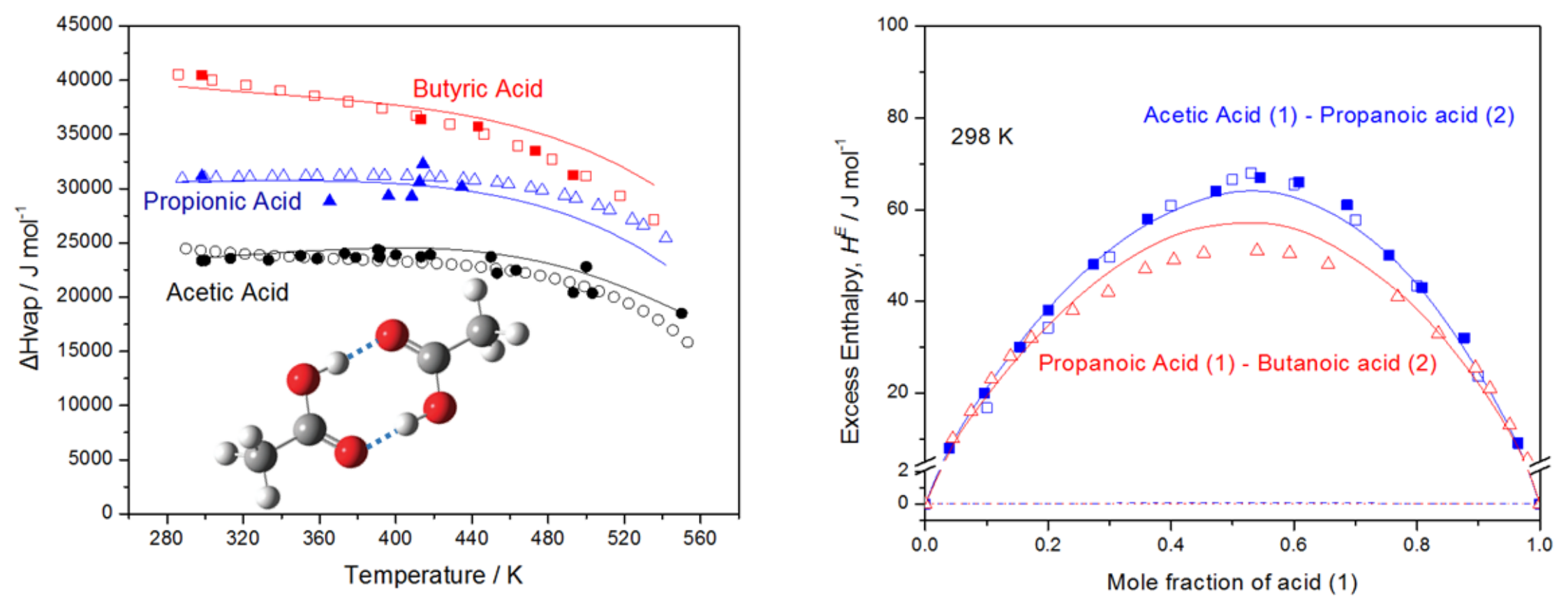